NUMBER THEORY WEEK 2017

BANACH CENTER PUBLICATIONS, VOLUME 118

INSTITUTE OF MATHEMATICS

POLISH ACADEMY OF SCIENCES

WARSZAWA 2019

\title{
SOME GENERATING FUNCTIONS OF THE RIEMANN ZETA FUNCTION
}

\author{
TAKUMI NODA \\ College of Engineering, Nihon University \\ 1 Nakagawara, Tokusada, Tamuramachi, Koriyama, Fukushima 963-8642, Japan \\ E-mail: takumi@ge.ce.nihon-u.ac.jp
}

\begin{abstract}
In this survey paper, functional relations between some generating functions of the Riemann zeta function are formulated. These generating functions themselves are zetafunctions (Bessel type, confluent-hypergeometric type and hypergeometric type), introduced by M. Katsurada and the author. The explicit formula of the special values of these zeta functions at non-positive integers are also given.
\end{abstract}

1. Introduction. In the generalization of Glaisher's formula [Gl]

$$
\gamma=1-2\left\{\frac{\zeta(3)}{3 \cdot 4}+\frac{\zeta(5)}{5 \cdot 6}+\frac{\zeta(7)}{7 \cdot 8}+\ldots\right\},
$$

Ramanujan [Ra employed the binomial type generating function of the Riemann zetafunction

$$
\zeta(s, 1+x)=\sum_{m=0}^{\infty} \frac{\Gamma(s+m)}{\Gamma(s) m !} \zeta(s+m)(-x)^{m},
$$

for $|x|<1$ and $s \in \mathbb{C} \backslash\{1\}$. Here $\gamma$ is Euler's constant, $\zeta(s)$ and $\zeta(s, x)$ are the Riemann zeta-function and the Hurwitz zeta-function respectively, and $\Gamma(s)$ is the Gamma function. A number of studies of the generalizations and applications of Ramanujan's identity (1) have been conducted (for details, refer to [SC]).

In recent works [No1, No2 we introduced some new zeta-functions, Bessel zetafunctions and a confluent hypergeometric zeta-function, where we derived integral representations, transformation formulas, power series expansions involving the Riemann zeta-function, and recurrence formulas. The $J$-Bessel zeta-function introduced in [No1]

2010 Mathematics Subject Classification: Primary 11M41; Secondary 11F11.

Key words and phrases: zeta-function, generating function, Poincaré series.

The paper is in final form and no version of it will be published elsewhere. 
appears in the Fourier series expansion of the Poincaré series attached to $S L(2, \mathbb{Z})$ by applying the inverse Mellin transform. This fact strongly suggested that our zeta-functions should have a kind of functional equations. In this article, we re-describe transformation formulas (functional equations) of the $J$-Bessel zeta-function and the confluent hypergeometric zeta-function in terms of zeta functions introduced by M. Katsurada. In [Kt], Katsurada initially defined Gauss' hypergeometric type and Kummer's confluent hypergeometric type generating functions of $\zeta(s)$, and obtained Mellin-Barnes type integral representations. We give explicit formulas of the special values of our zeta functions at non-positive integers.

More general zeta functions twisted by hypergeometric or Bessel functions are treated by Kaczorowski and Perelli [KP3, KP4]. They derived meromorphic continuations of these zeta-functions via the properties of the nonlinear twists obtained in [KP1, KP2].

Throughout this paper, $s$ is a complex variable, $J_{\nu}(z)$ the Bessel function of the first kind (cf. Er2, 7.2.1. (2)]), ${ }_{2} F_{1}(\underset{\gamma}{\alpha, \beta} ; z)$ and $F(\alpha ; \gamma ; z)$ denote Gauss' hypergeometric function and Kummer's confluent hypergeometric function of the first kind respectively (cf. [Er1, 2.1.3. (10), 6.5. (1)]). Let $\int_{-\infty}^{(0+)}$ denote integration over a Hankel contour, starting at negative infinity on the real axis, encircling the origin with a small radius in the positive direction, and returning to the starting point. We also use the Pochhammer symbol $(s)_{m}=\Gamma(s+m) / \Gamma(s)$.

\section{First main theorems}

Definition 2.1 ( No1] $)$. Let $\theta>0, \nu \in \mathbb{C}$. The $J$-Bessel zeta-function of order $\nu-1$ is defined by

$$
\zeta_{J-B}(s ; \nu-1 ; \theta):=\sum_{n=1}^{\infty} \frac{J_{\nu-1}(2 \sqrt{\theta n})}{n^{s+(\nu-1) / 2}} .
$$

This quantity is equal to $\mathbf{J}_{\nu-1}(s-1 ; \theta)$ in [No1, (1.1)]. By the estimates of the $J$-Bessel function, the Dirichlet series (2) converges absolutely in the region $\operatorname{Re}(s)>1$ when $\operatorname{Re}(\nu)>1 / 2$, and converges absolutely in the region $\operatorname{Re}(s)>\lfloor 3 / 2-\operatorname{Re}(\nu)\rfloor / 2$ when $\operatorname{Re}(\nu) \leq 1 / 2$. In the specific case when $\nu$ is an integer, $\zeta_{J-B}(s ; \nu-1 ; \theta)$ converges absolutely in the region $\operatorname{Re}(s)>1+(1-\nu) / 2$ (cf. [No1, Proposition 3.1]).

Definition 2.2 ([No2 $])$. Let $(\alpha, \gamma) \in \mathbb{C} \times(\mathbb{C} \backslash\{0,-1,-2, \ldots\})$, and assume $-2 \pi<\theta<0$. The confluent hypergeometric zeta-function of the first kind is defined by

$$
\zeta_{\text {conf. I }}(s ; \alpha, \gamma ; \theta):=\sum_{n=1}^{\infty} \frac{F(\alpha ; \gamma ; \theta n)}{n^{s}} .
$$

These zeta-functions have integral representations, and are previously unknown generating functions of the Riemann zeta-function.

TheOREM 2.3 (Integral representation, power series expansion [No1, Theorem 1.1]). The $J$-Bessel zeta-function has an integral representation

$$
\zeta_{J-B}(s ; \nu-1 ; \theta)=\frac{\theta^{s+(\nu-1) / 2} \Gamma(1-s)}{2 \pi i \Gamma(\nu)} \int_{-\infty}^{(0+)} \frac{u^{s-1} e^{\theta u}}{1-e^{\theta u}} F\left(1-s ; \nu ;-u^{-1}\right) d u,
$$


which provides a meromorphic continuation to the whole s-plane. Further, the power series expansion

$$
\zeta_{J-B}(s ; \nu-1 ; \theta)=\sum_{m=0}^{\infty} \frac{\theta^{(\nu-1) / 2}}{\Gamma(\nu+m) m !} \zeta(s-m)(-\theta)^{m},
$$

holds for $s \in \mathbb{C} \backslash\{1,2, \ldots\}$.

THEOREM 2.4 (Integral representation, power series expansion [No2, Theorem 1.1]). The Dirichlet series (3) converges absolutely for $\operatorname{Re}(\alpha+s)>1$, and has an integral representation

$$
\zeta_{\text {conf. I }}(s ; \alpha, \gamma ; \theta)=\frac{\Gamma(1-s)}{2 \pi i} \int_{-\infty}^{(0+)} \frac{u^{s-1} e^{u}}{1-e^{u}}{ }_{2} F_{1}\left(\begin{array}{c}
\alpha, 1-s \\
\gamma
\end{array} \frac{\theta}{u}\right) d u,
$$

which provides an analytic continuation to the whole s-plane. Here we choose the radius $r$ in the Hankel contour above so as $|\theta|<r<2 \pi$. Further, the power series expansion

$$
\zeta_{\text {conf. I }}(s ; \alpha, \gamma ; \theta)=\sum_{m=0}^{\infty} \frac{(\alpha)_{m}}{(\gamma)_{m} m !} \zeta(s-m) \theta^{m}
$$

holds for $s \in \mathbb{C} \backslash\{1,2, \ldots\}$.

Proof of Theorems 2.3 and 2.4. Key lemmas in the proofs of (4) and (6) are FourierMellin integrals of the confluent hypergeometric function and Gauss' hypergeometric function, which are respectively equivalent to the inverse integral transform of Weber's first exponential integral (cf. Wa 13.3]) and the inverse Laplace transform of confluent hypergeometric function (cf. [Er1, 6.10. (5)]). For details, see [No1, Lemma 2.2] and [No2, Proposition 2.2]. The power series expansions (5) and (7) follow from Kummer's series (cf. [Er1, 6.1. (1)]) and the hypergeometric series (cf. [Er1, 2.1.1.]).

3. Functional relations. In [Kt], Katsurada introduced the following two generating functions of $\zeta(s)$, and derived some integral representations.

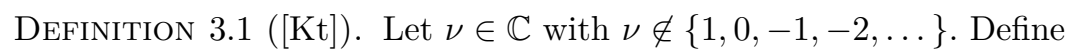

$$
\begin{array}{rlr}
\mathcal{F}_{\nu}(\alpha, \beta ; \gamma ; z) & :=\sum_{m=0}^{\infty} \frac{(\alpha)_{m}(\beta)_{m}}{(\gamma)_{m} m !} \zeta(\nu+m) z^{m} & (|z|<1), \\
\mathcal{F}_{\nu}(\alpha ; \gamma ; z) & :=\sum_{m=0}^{\infty} \frac{(\alpha)_{m}}{(\gamma)_{m} m !} \zeta(\nu+m) z^{m} & (|z|<+\infty)
\end{array}
$$

for $(\alpha, \beta) \in \mathbb{C}^{2}$ and $\gamma \in \mathbb{C} \backslash\{0,-1,-2, \ldots\}$.

Substituting $\zeta(\nu+m)=\sum_{n=1}^{\infty} n^{-\nu-m}$ for $\operatorname{Re}(\nu)>1$ and $m \geq 0$, we see immediately Theorem 3.2 ([ $\mathrm{Kt}$, Theorem 5.1.]). The Dirichlet series expressions

$$
\mathcal{F}_{\nu}(\alpha, \beta ; \gamma ; z)=\sum_{n=1}^{\infty}{ }_{2} F_{1}\left(\begin{array}{c}
\alpha, \beta \\
\gamma
\end{array} \frac{z}{n}\right) n^{-\nu}, \quad \mathcal{F}_{\nu}(\alpha ; \gamma ; z)=\sum_{n=1}^{\infty} F\left(\alpha, \gamma ; \frac{z}{n}\right) n^{-\nu}
$$

hold for $\operatorname{Re}(\nu)>1$.

Now we describe functional relations (functional equations) of $\zeta_{J-B}(s ; \nu-1 ; \theta)$ and $\zeta_{\text {conf. I }}(s ; \alpha, \gamma ; \theta)$ in terms of Katsurada's generating functions. 
Main Theorem 3.3 (Functional relations). For $s \in \mathbb{C} \backslash\{0,1,2, \ldots\}$, we have

$$
\begin{aligned}
\zeta_{J-B}(s ; \nu-1 ; \theta) \\
\quad=\frac{\theta^{(\nu-1) / 2} \Gamma(1-s)}{(2 \pi i)^{1-s} \Gamma(\nu)}\left\{\mathcal{F}_{1-s}\left(1-s ; \nu ; \frac{-\theta}{2 \pi i}\right)+(-1)^{s-1} \mathcal{F}_{1-s}\left(1-s ; \nu ; \frac{\theta}{2 \pi i}\right)\right\},
\end{aligned}
$$

and

$\zeta_{\text {conf. I }}(s ; \alpha, \gamma ; \theta)$

$$
=\frac{\Gamma(1-s)}{(2 \pi i)^{1-s}}\left\{\mathcal{F}_{1-s}\left(\alpha, 1-s ; \gamma ; \frac{\theta}{2 \pi i}\right)+(-1)^{s-1} \mathcal{F}_{1-s}\left(\alpha, 1-s ; \gamma ; \frac{-\theta}{2 \pi i}\right)\right\} .
$$

Proof. By shifting the paths of integrals (4) and (6), we have transformation formulas

$$
\begin{gathered}
\zeta_{J-B}(s ; \nu-1 ; \theta)=\frac{\theta^{(\nu-1) / 2} \Gamma(1-s)}{\Gamma(\nu)} \sum_{n=-\infty, n \neq 0}^{\infty}(2 \pi i n)^{s-1} F\left(1-s ; \nu ; \frac{-\theta}{2 \pi i n}\right), \\
\zeta_{\text {conf. I }}(s ; \alpha, \gamma ; \theta)=\Gamma(1-s) \sum_{n=-\infty, n \neq 0}^{\infty}(2 \pi i n)^{s-1}{ }_{2} F_{1}\left(\begin{array}{c}
\alpha, 1-s \\
\gamma
\end{array} ; \frac{\theta}{2 \pi i n}\right)
\end{gathered}
$$

for $\operatorname{Re}(s)<0$ (see [No1, Theorem $1.1(1.4)]$ and [No2, Theorem $1.1(1.4)]$ ). Substituting (8) into (11) and (12), we obtain the functional relations $(9)$ and (10), which are analytically continued to the whole $s$-plane by Definition 3.1

Corollary 3.4 (Special values at non-positive integers). Let $k=0,1,2, \ldots$ and let $B_{m}$ denote the $m$-th Bernoulli number. Then

$$
\begin{aligned}
& \zeta_{J-B}(-k ; \nu-1 ; \theta)=\sum_{m=0}^{\infty} \frac{(-1)^{-k} \theta^{(\nu-1) / 2+m}}{(1+k+m) \Gamma(\nu+m) m !} B_{1+k+m}, \\
& \zeta_{\text {conf. I }}(-k ; \alpha, \gamma ; \theta)=\sum_{m=0}^{\infty} \frac{(-1)^{-k-m} \theta^{m}(\alpha)_{m}}{(1+k+m) m !(\gamma)_{m}} B_{1+k+m} .
\end{aligned}
$$

Proof. Using $\zeta(-k)=(-1)^{k} B_{1+k} /(1+k)$ for $k=0,1,2, \ldots$ (cf. [Ti, (2.4.3)]) in (5) and (7) we obtain the assertions of Corollary 3.4

Corollary 3.4 can also be proved via $(9)$ and $(10$ when $k=1,2, \ldots$ In the following, we show some examples of the special value of $\zeta_{J-B}(s ; \nu-1 ; \theta)$ at non-positive integers. EXAMPLE 3.5.

(i) For $s=0, \nu=1, \theta=1$, we have $\zeta_{J-B}(0 ; 0 ; 1)=\sum_{m=0}^{\infty} \frac{B_{m+1}}{(m+1)(m !)^{2}}$,

(ii) For $s=-1, \nu=1, \theta=1$, we have $\zeta_{J-B}(-1 ; 0 ; 1)=\sum_{m=0}^{\infty} \frac{-B_{m+2}}{(m+2)(m !)^{2}}$,

(iii) For $s=0, \nu=\frac{3}{2}, \theta=4$, we have $\zeta_{J-B}\left(0 ; \frac{1}{2} ; 4\right)=\frac{1}{\sqrt{2 \pi}} \sum_{m=0}^{\infty} \frac{B_{m+1}}{(m+1)(2 m+1) !}$.

Acknowledgments. This work was partially supported by JSPS KAKENHI Grant Number JP16K05078. 


\section{References}

[Er1] A. Erdélyi, W. Magnus, F. Oberhettinger, F. G. Tricomi, Higher Transcendental Functions, Vol. I, McGraw-Hill, New York, 1953.

[Er2] A. Erdélyi, W. Magnus, F. Oberhettinger, F. G. Tricomi, Higher Transcendental Functions, Vol. II, McGraw-Hill, New York, 1953.

[Gl] J. W. L. Glaisher, Relations connecting quantities of the form $1+2^{-n}+3^{-n}+4^{-n} \&$ c., Messenger Math. 44 (1915), 1-10.

[KP1] J. Kaczorowski, A. Perelli, On the structure of the Selberg class, I: $0 \leq d \leq 1$, Acta Math. 182 (1999), 207-241.

[KP2] J. Kaczorowski, A. Perelli, On the structure of the Selberg class, VI: Non-linear twists, Acta Arith. 116 (2005), 315-341.

[KP3] J. Kaczorowski, A. Perelli, A note on Bessel twists of L-functions, in: Analytic Number Theory, Springer, Cham, 2015, 195-200.

[KP4] J. Kaczorowski, A. Perelli, Twists and resonance of L-functions, I, J. Eur. Math. Soc. (JEMS) 18 (2016), 1349-1389.

[Kt] M. Katsurada, On Mellin-Barnes type integrals and sums associated with the Riemann zeta-function, Publ. Inst. Math. (Beograd) (N.S.) 62(76) (1997), 13-25.

[No1] T. Noda, On the functional properties of Bessel zeta-functions, Acta Arith. 171 (2015), $1-13$.

[No2] T. Noda, On the functional properties of the confluent hypergeometric zeta-function, Ramanujan J. 41 (2016), 183-190.

[Ra] S. Ramanujan, A series for Euler's constant $\gamma$, Messenger Math. 46 (1917), 73-80.

[SC] H. M. Srivastava, J. Choi, Zeta and q-Zeta Functions and Associated Series and Integrals, Elsevier, Amsterdam, 2012.

[Ti] E. C. Titchmarsh, The Theory of the Riemann Zeta-Function, 2nd ed. edited by D. R. Heath-Brown, Clarendon Press, New York, 1986.

[Wa] G. N. Watson, A treatise on the theory of Bessel functions. Cambridge Univ. Press, Cambridge, 1922. 
